\title{
Boosted learned kernels for data-driven vesselness measure
}

\author{
E. Grisan \\ Department of Information Engineering, , University of Padova, Padova, Italy
}

\begin{abstract}
Common vessel centerline extraction methods rely on the computation of a measure providing the likeness of the local appearance of the data to a curvilinear tube-like structure. The most popular techniques rely on empirically designed (hand crafted) measurements as the widely used Hessian vesselness, the recent oriented flux tubeness or filters (e.g. the Gaussian matched filter) that are developed to respond to local features, without exploiting any context information nor the rich structural information embedded in the data.

At variance with the previously proposed methods, we propose a completely data-driven approach for learning a vesselness measure from expert-annotated dataset. For each data point (voxel or pixel), we extract the intensity values in a neighborhood region, and estimate the discriminative convolutional kernel yielding a positive response for vessel data and negative response for non-vessel data. The process is iterated within a boosting framework, providing a set of linear filters, whose combined response is the learned vesselness measure.

We show the results of the general-use proposed method on the DRIVE retinal images dataset, comparing its performance against the hessian-based vesselness, oriented flux antisymmetry tubeness, and vesselness learned with a probabilistic boosting tree or with a regression tree. We demonstrate the superiority of our approach that yields a vessel detection accuracy of 0.95 , with respect to 0.92 (hessian), 0.90 (oriented flux) and 0.85 (boosting tree).
\end{abstract}

Keywords: vesselness, retina, oriented flux, filter learning, vessel detection, vessel segmentation

\section{INTRODUCTION}

The enhancement and detection of curvilinear tube-like structures, ranging from blood vessels to nerves and dendrites, is becoming a crucial step for the evaluation and diagnosis of different pathologies, under different imaging modalities (CT, MRI, optical microscopy, angiography). The inherent variability of the curvilinear structures, either due to pathophysiological variability, or to imaging artifacts, or to contrast and intensity variations, make the problem extremely difficult. Most segmentation methods use an empirical (although principled) formulation of a vesselness measure, i.e. a measure of how well the local appearance of the data match the appearance of an ideal tube-like structure imaged under similar conditions: we will refer to them as hand-crafted. The most widespread measure of vesselness assume that blood vessels can be considered as elongated or line structures, of different diameters and orientations, either dark on a brighter background (as in the retinal images), or bright on a darker background (as in CT angiography). Moreover, vessels are assumed to be characterized by a local principal direction and a cross-sectional plane (in 3D) or line (in 2D) with Gaussian intensity profile. Under these assumptions, the most common tool to characterize vessel patterns is through the Hessian matrix. ${ }^{1-3}$ In order to mitigate some of the limitations of the Hessian-based measures, a family of vesselness measures based on the oriented flux, have have been recently proposed. ${ }^{4,5}$ These methods provide successful tubularity measures, for their supposed ability in detecting curvilinear structures, resilience to adjacent confounders and robustness to noise. However, no context information is accounted for in these measures, nor they are built to exploit all information embedded in the available data. In order to capture these additional characteristics, machine learning approaches have been proposed to provide a detection score that can be treated as a vesselness measurement. ${ }^{6,7}$ However, they are based on the extraction of user-defined features that are only supposed to capture the discriminant characteristics of vessels and non-vessels.

Building on the idea that the score provided by a supervised classifier may be used as a vesselness measure, and at variance with the hybrid approach proposed in ${ }^{8}$ we propose a fully data-driven approach, in which the features

Further author information: (Send correspondence to enrico.grisan@dei.unipd.it 
to be used with an AdaBoost classifier are learned as optimal linear filters from the training set. By this means, there is no need of any preliminary feature extraction and the testing stage requires only the computation of convolutions of the learned filters with the data and the linear combinations of their responses.

\section{BOOSTED VESSELNESS KERNEL (BVK)}

Following the approach proposed in $^{9}$ we estimate a set of discriminative convolution kernels (or linear weak learners) allowing a linear classification of each pixel into the two classes of interest through AdaBoost, making the most use of the local appearance information, and then, as in, ${ }^{7}$ we use the classifier output as vesseleness measure. Since we are dealing with a problem in which the geometric information is weak, even referencing the position of each pixel with respect to the optic disk, we choose to evaluate image features alone.

If we are given a set of $N$ observations $\mathbf{f}_{1}, \ldots, \mathbf{f}_{N} \in \mathbb{R}^{d}$ and labels $l_{1}, \ldots, l_{N} \in\{-1,+1\}$, we want to design a function $H: \mathcal{F} \mapsto\{-1,+1\}$, a classifier, that predicts the label $l$ of a generic observation $\mathbf{f}$. As the observations $\mathbf{f}_{i} \in \mathbb{R}^{d}$ we are dealing with are image regions, $d$ is the number of pixels in a $\sqrt{d} \times \sqrt{d}$ region. We denote the unknown joint probability distribution density of $(\mathbf{f}, l)$ as $p(\mathbf{f}, l)$ and the expectation of a random variable $w$ with respect to $p$ as $E_{p}[w]$. The unknown probability density $p(f, l)$ can be written as a Parzen window estimate with a Gaussian kernel $G_{\sigma}(\cdot)$ with zero-mean and standard deviation $\sigma$, resulting in the smoothed expected risk

$$
\operatorname{Err}_{\hat{p}_{g}}(H)=-\frac{1}{N} \sum_{i=1}^{N} \int G_{\sigma}\left(\mathbf{f}-\mathbf{f}_{i}\right) l_{i} H(\mathbf{f}) d \mathbf{f} .
$$

A classifier $H$ is designed by additively and linearly combining several weak classifiers $h_{m}(\mathbf{f})$, each of them doing at least better than the chance. ${ }^{10}$ If we consider $M$ weak classifiers $h_{m}: \mathbb{R}^{2} \mapsto\{-1,1\}$, and a set of $M$ scalar parameters $\alpha_{1}, \ldots, \alpha_{M} \in \mathbb{R}$, we can build a strong vesselness estimator as:

$$
V_{M}(\mathbf{f})=\sum_{m=1}^{M} \alpha_{m} G_{\sigma} * h_{m}(\mathbf{f}) .
$$

Restricting $h_{m}$ to be computed as the sign of a scalar product $h_{m}(\mathbf{f})=\operatorname{sign}\left(\gamma_{0}+\gamma_{1} \cdot \mathbf{f}\right)$, with $\gamma_{0} \in \mathbb{R}$ and $\gamma_{1} \in \mathbb{R}^{d}$, the parameters $\gamma_{0}$ and $\gamma_{1}$ can be estimated by minimizing via gradient descent the empirical error:

$$
\operatorname{Err}_{\hat{p}_{g}}\left(h_{m}\right)=\frac{1}{2}-\frac{1}{2 N} \sum_{i=1}^{N} w_{i, m} l_{i} \operatorname{erf}\left(\frac{\gamma_{0, m}+\gamma_{1, m} \cdot \mathbf{f}}{\sqrt{\gamma_{1, m}^{T} \Sigma \gamma_{1, m}}}\right)
$$

with $w_{i}$ the weight of the training sample $\left(\mathbf{f}_{i}, l_{i}\right)$, and $\operatorname{erf}(\cdot)$ is the error function. In practice, each estimated $\gamma_{1, m}$ can be rearranged as an image and interpreted as a vesselness filter kernel, providing as output a weak-vesselness measure, that is combined with other kernels to provide the strong vesselness measure $V_{M}$. In our approach, the single weak-vesselness kernel i sequentially estimated within an AdaBboost framework. By this means, the boosting is performed by initializing the weight $w_{i, 1}=1 / N$ in Eq. 3 . Then, the algorithm to boost learned filter is described below.:

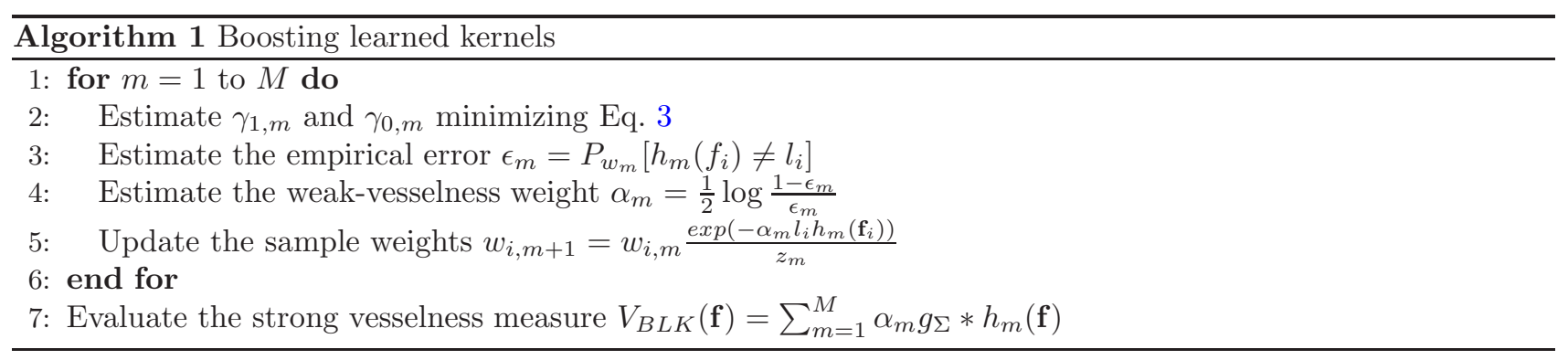




\section{RELATED METHODS}

\subsection{Hand crafted vesselenss measures}

\subsubsection{Hessian-based vesselness (HES)}

Starting from a scale-space representation of an image $I(x, y), L(x, y ; t)=G_{\sigma}(x, y) * I(x, y)$, where $G_{\sigma}(\cdot)$ is a Gaussian function with variance $\sigma$, the larger eigenvalue $\lambda_{1}$ and the smaller eigenvalue $\lambda_{2}$ of the Hessian matrix can be computed. $\operatorname{In}^{2}$ the ridgeness score $R(x, y)=\lambda_{2} / \lambda_{1}$ and the Frobenius norm of the Hessian $S(x, y)$ are combined to provide a vesselness measure at the position $(x, y)$ for scale $t$ :

$$
v_{\text {hess }}(x, y ; t)=\left\{\begin{array}{cc}
0 & \lambda_{1}>0 \\
\exp \left(-\frac{R^{2}}{2 \beta^{2}}\right)\left(1-\exp \left(-\frac{S^{2}}{2 c^{2}}\right)\right) & \text { otherwise. }
\end{array}\right.
$$

The final vesselness measure $V(x, y)$ across the different scales is computed as the maximum response $V_{\text {hess }}(x, y)=$ $\max _{t} v_{\text {hess }}(x, y ; t)$.

\subsubsection{Optimally Oriented Flux tubeness (OOF)}

The Optimally Oriented Flux (OOF) filter is computed by convolving the second derivatives of the image with the indicator function of the sphere. ${ }^{4}$ Given a position $(x, y)$ in the image $I$, a radius $r$, and and orientation $p$, the OOF filter response $f(x, y ; r, p)$ is obtained by integrating the projected image gradients in the $p$ direction over a sphere $\partial S_{r}$ of radius $r$ centered at $(x, y)$ :

$$
f(x, y ; r, p)=\frac{1}{4 \pi r^{2}} \int_{\partial S_{r}}\left(\nabla\left(G_{\sigma} * I\left(x+r n_{x}, y+r n_{y}\right)\right) \cdot p\right) p \cdot n d S,
$$

where $n=\left(n_{x}, n_{y}\right)$ is the outward versor normal to $\partial S_{r}, G_{\sigma}$ is a smoothing Gaussian function with standard deviation equal to $\sigma$, and $d S$ is the infinitesimal area element of the sphere $\partial S_{r}$. The idea of enhancing vessel structures through $f(x, y ; r, p)$, is that the smaller its value, the more likely is that the position $(x, y)$ is the center of a vessel with scale $r$ and orientation $p$. Hence, the OOF tubeness measure can be computed as: ${ }^{4}$

$$
f_{O O F}(x, y ; r)=\max _{p_{1}, p_{2} \mid p_{1} \perp p_{2}}-f\left(x, y ; p_{1}, r\right)-f\left(x, y ; p_{2}, r\right) .
$$

In order to prevent a strong response of $f_{O F}(x, y ; r)$ to the edges, the optimally oriented flux has been extended ${ }^{5}$ with an antisymmetry function correction term, to obtain an oriented flux antisymmetry (OFA) tubeness $v_{O F A}(x, y ; r)$, that yields high responses along centerlines of curvilinear structures at their associated scales (as the $f_{O O F}$ measure), at the same time annihilating the OOF response away from the structure centerlines:

$$
V_{O F A}(x, y)=\max _{r} v_{O F A}(x, y ; r) \text {. }
$$

\subsection{Learned probabilistic boosted tree (PBT)}

$\mathrm{In}^{7}$ a learning-based vesselness measurement has been proposed for 3D CT coronary data, based on a classifier that assigns a high score to voxels inside the vessels and a low score to those outside, making use of both position features (in this case with respect to the heart reference), and image features. Given a point at coordinates $(x, y)$ with intensity $I(x, y)$ and gradient $G(x, y)=\left(G_{x}, G_{y}\right)=\left(\nabla_{x} I, \nabla_{y} I\right)$, and the angle between the gradient $G$ and the y axis as $\alpha=\arccos \left(G_{y}\right), 24$ local features based on the image intensity and gradient are extracted. The features to be used are $I, \sqrt{I}, \sqrt[3]{I}, I^{2}, I^{3}, \log I,\|G\|, \sqrt{\|G\|}, \sqrt[3]{\|G\|},\|G\|^{2},\|G\|^{3}, \log \|G\|, \alpha, \sqrt{a l p h a}, \sqrt[3]{\alpha}$, $\alpha^{2}, \alpha^{3}, \log \alpha, G_{x}, G_{y}$. In total, there are 20 local features for each sampling point, based on intensity, gradient and gradient orientation, that are sampled in the $5 \times 5$ neighborhood of the position under analysis, resulting in a total of $25 \times 24$ image features. These features are then fed to a trained probabilistic boosting tree $(\mathrm{PBT})^{11}$ to classify a pixel as inside a retinal vessel or belonging to the retinal background, providing as output the vesselness measure $V_{P B T}$. 


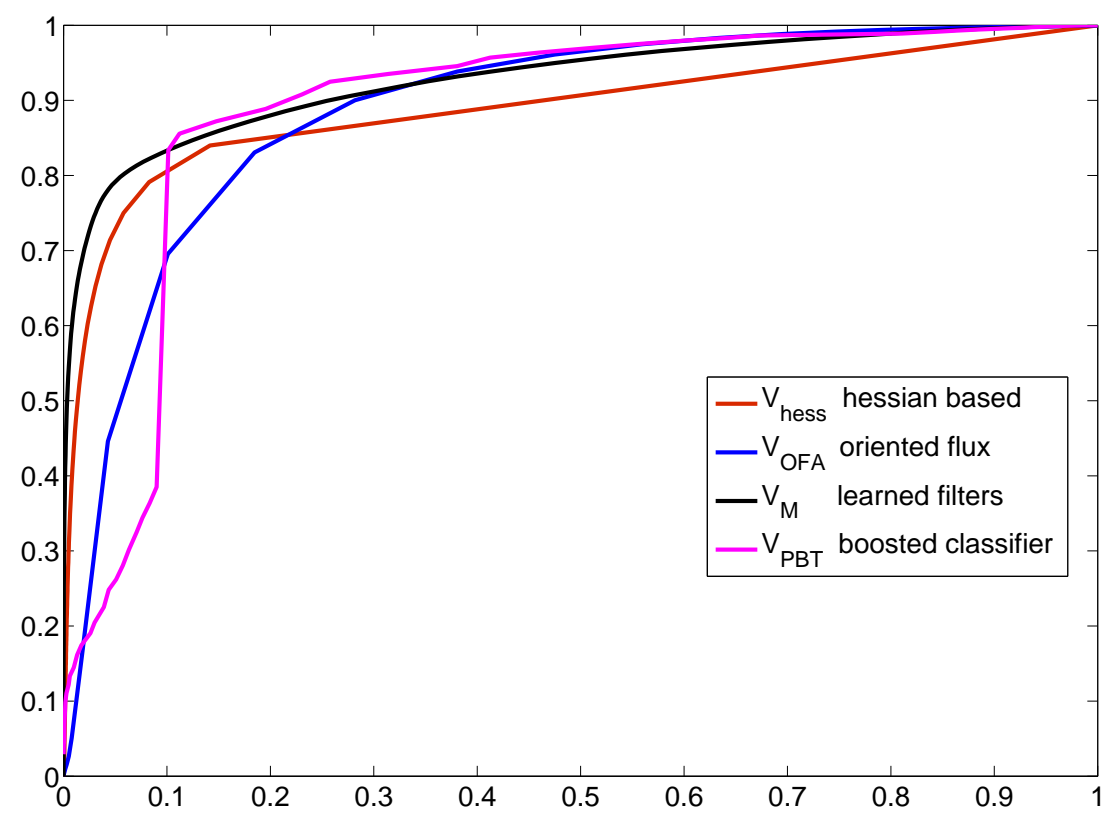

Figure 1. ROC curves obtained by thresholding the different vesselness measures. On the x-axis the fraction of false positive pixels (1-specificity) and on the $y$-axis the fraction of true positive pixels (sensitivity).

\section{EXPERIMENTS AND RESULTS}

We applied the proposed procedure to the DRIVE ${ }^{12}$ data set, learning the kernels on the training set composed by 20 retinal fundus images, with corresponding manual annotation of retinal vessels. The additional 20 images in the DRIVE dataset for testing were used for evaluating the vesselness measures. For training the proposed boosted kernels, a random subset of pixels from the greem plane of the training images ( $1.3 \%$ of the total) was extracted after normalizing the image ${ }^{13}$ to obtain a uniform appearance and reduce inter-image variability. For each selected pixel $\left(x_{i}, y_{i}\right)$, we defined $f_{i}$ as the square window neighborhood of $15 \times 15$ pixels centered on the selected pixel, with a 3 -scale pyramidal representation, so that $f_{i}$ is a real valued vector with $225 \mathrm{x} 1$ elements. In order to reduce the intra-class variability of $f_{i}$, each $15 \mathrm{x} 15$ window was preventively rotated so as to have the vessel horizontally oriented.

The method proposed in $^{7}$ were trained using a probabilistic boosted tree (PBT) with depth set to 3 and with 20 weak-learners for each node, on the same features described in the original paper, with the exception of those providing the localization of the pixel with respect to the heart (that would be translated to reference retinal structures).

Testing was performed convolving the test image with $R$ different rotated versions of each estimated $h_{m}$ (at the orientations $r \pi / R$ radians, with $r=0, R-1)$, obtaining then $\mathrm{R}$ different $V_{M}\left(x_{i} ; r\right)$. The estimated vesselness for each pixel $(x, y)$ is then simply $V_{M}(x, y)=\max _{r} V_{M}(x, y ; r)$. The same training and testing procedure has been performed for the machine-learning vesselness of, ${ }^{7}$ whereas for the hessian-based and OFA vesselness measure, their value has been computed for each pixel of each image. Representative results of the vesselness output for the different methods are reported in Fig. 2. When the four methods are operated with the best respective threshold estimted through a ROC analysis, they provide a mean vessel detection accuracy of 0.90 for OFA, 0.92 for HES, 0.85 for PBT, and 0.95 for BLK.

Representative results of the vesselness output for the different methods are reported in Fig. 2, whereas the ROC curves of the four methods for vessel detection on the retinal 20 retinal images used is shown in Fig. 1. The curve of the proposed method is mostly above all others, demonstrating its better ability in discriminating 
(even with a small number of weak-learners) vessel pixels from non-vessel pixels. When the four methods are operated with the best respective threshold, they provide a mean vessel detection accuracy of 0.90 for $V_{O O F}$, 0.92 for $V_{H E S}, 0.90$ for $V_{P B T}, 0.94$ for $V_{L R T}$, and 0.95 for $V_{B} K$.

\section{CONCLUSIONS}

We present a very general method for estimating data-driven boosted filters that proved to be able to effectively learn the discriminative characteristics of vessels and non-vessels data. In the case study of retinal vessels enhancement and detection, the proposed method outperformed both the hessian-based vesselness enhancement, the asymmetry oriented flux and the feature based PBT. The proposed method has the advantage of being modular, since weak classifiers can be added in a greedy fashion thanks to the underlying AdaBoost, with low computational complexity at training time and requiring only a set of convolution at testing time. Since it learns discriminative linear kernels from the raw data (or from some preprocessing of them), it can be extended straightforwardly to higher dimensional data with no increase in complexity.

\section{REFERENCES}

[1] Sato, Y., Nakajima, S., Atsumi, H., Koller, T., Gerig, G., Yoshida, S., and Kikinis, R., "3d multi-scale line filter for segmentation and visualization of curvilinear structures in medical images," in [Proc. CVRMedMRCAS 1997], 213222 (1997).

[2] Frangi, A. F., Niessen, W. J., Vincken, K. L., and Viergever, M. A., "Multiscale vessel enhancement filtering," in [Medical Image Computing and Computer-Assisted Intervention - MICCAI 98], 130-137 (1998).

[3] Salem, N. and Nandi, A., "Unsupervised segmentation of retinal blood vessels using a single parameter vesselness measure," in [Sixth Indian Conference on Computer Vision, Graphics \& Image Processing, 2008. ICVGIP '08.], 528-534 (2008).

[4] Law, M. W. K. and Chung, A. C. S., "Efficient implementation for spherical flux computation and its application to vascular segmentation," IEEE Transactions on Image Processing 18(3), 596612 (2009).

[5] Law, M. and Chung, A., "An oriented flux symmetry based active contour model for three dimensional vessel segmentation," in [ECCV 2010], 720734 (2010).

[6] Lupascu, C., Tegolo, D., and Trucco, E., "Fabc: retinal vessel segmentation using adaboost.," IEEE Trans Inf Technol Biomed. 14(09), 1267-1274 (2010).

[7] Zheng, Y., Loziczonek, M., Georgescu, B., Zhou, S. K., Vega-Higuera, F., and Comaniciu, D., "Machine learning based vesselness measurement for coronary artery segmentation in cardiac ct volumes," in [Proc. SPIE 7962, Medical Imaging], (2011).

[8] Annunziata, R., Kheirkhah, A., Hamrah, P., and Trucco, E., "Combining efficient hand-crafted features with learned filters for fast and accurate corneal nerve fibre centreline detection," in [IEEE International Conference on Engineering in Medicine and Biology, EMBC 2015], 5655-5658 (2015).

[9] Vedaldi, A., Favaro, P., and Grisan, E., "Boosting invariance and efficiency in supervised learning," in [IEEE International Conference on Computer Vision - ICCV 2007], (2007).

[10] Friedman, J., Hastie, T., and Tibshirani, R., "Additive logistic regression: a statistical view of boosting," The annals of Statistics 28, 337-407 (2000).

[11] Tu, Z., "Probabilistic boosting-tree: Learning discriminative models for classification, recognition, and clustering," in [Proc. of the Internationall Conference on Computer Vision, ICCV], 1589-1596 (2005).

[12] Staal, J., Abramoff, M., Niemeijer, M., Viergever, M., and von Ginneken, B., "Ridge based vessel segmentation in color images of the retina," IEEE Transactions on Medical Imaging 23(4), 501-509 (2004).

[13] Foracchia, M., Grisan, E., and Ruggeri, A., "Luminosity and contrast normalization in retinal images," Medical Image Analysis 9(3), 179-190 (2005). 


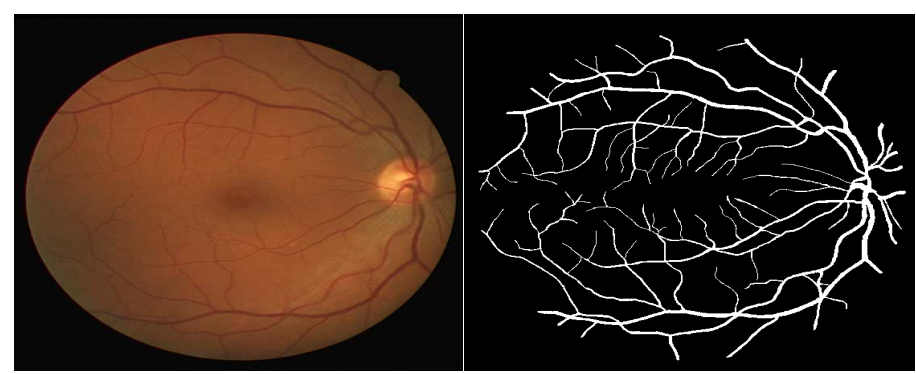

(a)

(b)

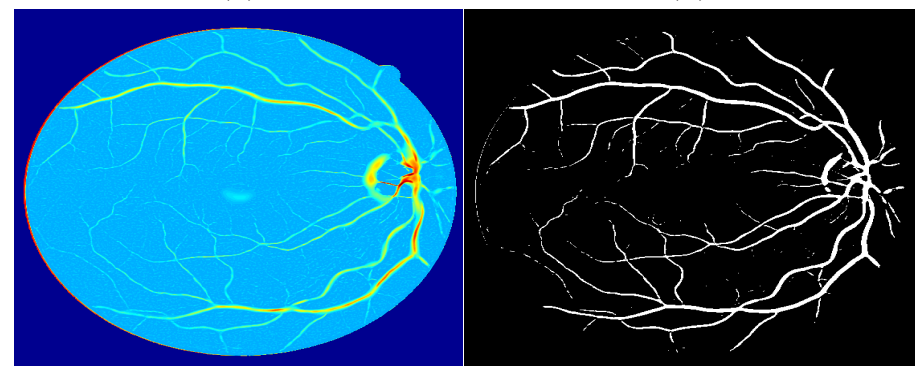

(c)

(d)

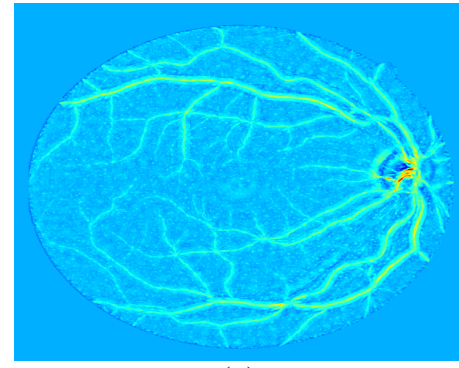

(e)

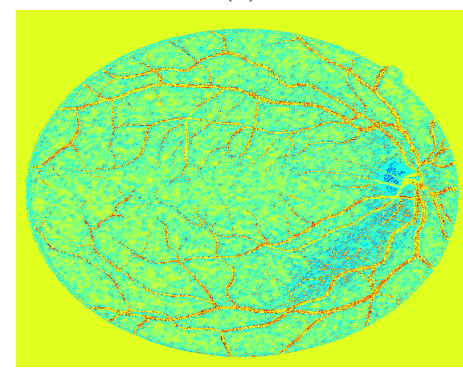

(g)

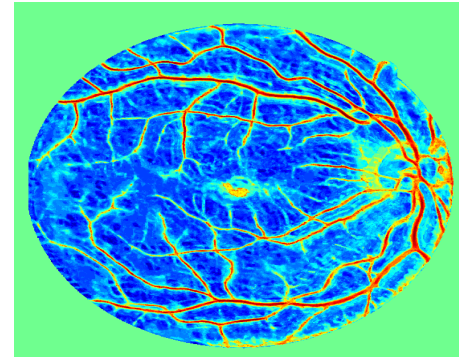

(i)

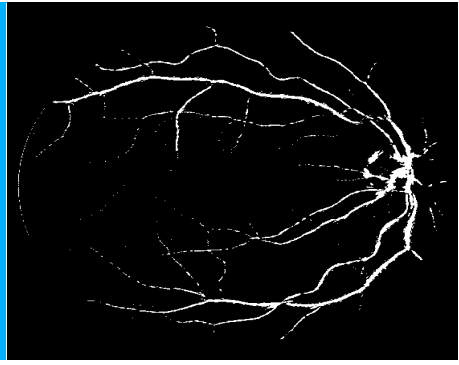

(f)

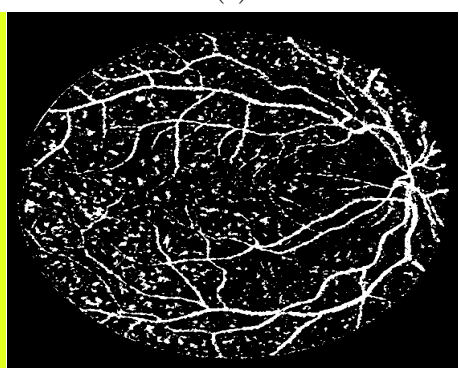

(h)

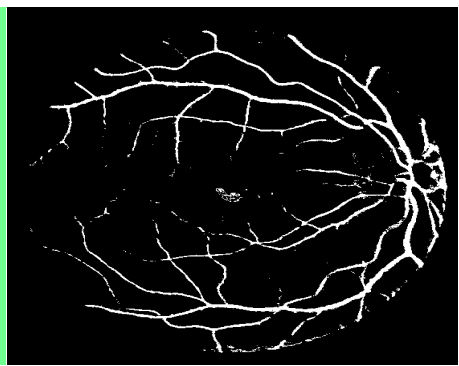

(j)

Figure 2. Vesselness measure on a random image of the DRIVE retinal dataset. Top row, the original image and the vesselness maps obtained with the the hessian-based method $V_{h e s s},{ }^{2}$ the oriented flux $V_{O F A},{ }^{5}$ the learned vesselness on image and gradient features $V_{P B T^{7}}$ and the proposed boosted learned vesselness. Bottom row, the corresponding vessel segmentation obtained by thresholding the vesselness map. 